\title{
Teaching Corner: The management of immune thrombocytopenic purpura
}

\section{BI Chosamata}

Department of Pathology and Laboratory Science, College of Medicine, University of Malawi, Blantyre, Malawi.

E-mail: bchosamata@medcol.mw

\section{Introduction}

Thrombocytopenia, defined as a platelet count less than 100 $\mathrm{x} 10^{9} / \mathrm{L}$, is common in clinical practice and poses diagnostic and management difficulties.

Haemostasis involves a delicate balance between procoagulant and anticoagulant mechanisms. The major components of haemostasis are platelets, coagulation factors, coagulation inhibitors, fibrinolysis and blood vessels. ${ }^{1}$ In severe thrombocytopenia, this haemostatic balance may be disturbed, leading to bleeding. Bleeding caused by thrombocytopenia is mucocutaneous and can present in different forms, which may include epistaxis, menorrhagia, petechiae, ecchymosis, subconjuctival haemorrhage and oozing of blood from surgical incision sites.

Qualitative platelet disorders can also cause mucocutaneous bleeding. These qualitative platelet disorders can be hereditary or acquired. Acquired causes include uraemia in chronic renal disease and the antiplatelet action of drugs, such as aspirin. ${ }^{2}$ The pattern of bleeding caused by coagulation disorders is quite different from that caused by platelet abnormalities. Bleeding resulting from coagulation disorders is characterised by bleeding into the joints, muscles, and connective tissue. ${ }^{1}$ Definitive diagnosis of qualitative platelet dysfunction cannot be made in Malawi; in our context, especially, the importance of a good clinical history cannot be overstated.

Quantitative platelet disorders can be easily diagnosed from a full blood count that reveals thrombocytopenia. One cause of thrombocytopenia is immune thrombocytopenic purpura (ITP), and this will be the topic of discussion in this scholarly corner paper.

\section{Mechanism of ITP}

ITP is a heterogeneous disease characterised by increased platelet clearance and insufficient platelet production. Increased platelet destruction takes place in the tissue macrophages, predominantly in the spleen. Several features suggest the destruction of platelets is mediated by the immunoglobulin fraction, $\operatorname{IgG}$ in particular, in the reticuloendothelial system. There is also inhibition of platelet release by the platelet-making cells (megakaryocytes) in the bone marrow. ${ }^{3,4}$

What causes the initial development of these antiplatelet antibodies is not clear, however sometimes a potential underlying cause, such as infection with hepatitis C, HIV, or Helicobacter pylori, is identified in some patients with immune thrombocytopenia. ${ }^{4,5}$

Platelet production is stimulated by thrombopoietin (TPO), which is secreted by the liver. In normal circumstances, the amount of free TPO released into the circulation is constant at all times. As TPO molecules bind to TPO receptors on haemopoietic stem cells and megakaryocytes, they are internalized, degraded, and removed from the circulation. The levels of free TPO are regulated by the number of circulating platelets and the megakaryocyte mass. When platelet counts are low, excess free TPO is available for binding to megakaryocytes, causing an increase in thrombopoiesis; and when platelet counts are high, less free TPO is available for binding. TPO levels are usually normal or slightly decreased in ITP and not high, as might be expected. ${ }^{6,7}$ Normal TPO levels in the context of ITP may be explained by the rapid clearance of TPO from the circulation that results from increased platelet turnover (due to the presence platelet antibodies). In ITP, the number of megakaryocytes is normal (and not increased) on bone marrow examination.

Patients with ITP usually present with isolated thrombocytopenia. White cell count is usually normal, and haemoglobin is also normal, unless there has been significant blood loss related to the thrombocytopenia.

\section{HIV-associated thrombocytopenia}

Although mild to moderate thrombocytopenia is common in patients with HIV, it is rarely associated with bleeding. The main causes of this thrombocytopenia are opportunistic infections, splenomegaly, and fever, which lead to decreased survival of platelets. ${ }^{8}$ Platelet-associated antibodies increase in prevalence with HIV progression. These antibodies consist of anti-glycoprotein II/Ia antibody. Some of these antibodies bind epitopes with homology to HIV-1 proteins nef, gag, env, and pol, suggesting molecular mimicry. ${ }^{8}$ These antibodies are present on the surface of platelets in conditions such as sepsis and thrombotic thrombocytopenic purpura (TTP).

These platelet-associated antibodies deposit themselves on the platelet surface and promote early platelet removal by the liver and spleen. ${ }^{9}$

Forpatients with advanced HIV disease, the thrombocytopenia is mainly caused by bone marrow failure. Defective megakaryopoiesis may also contribute to thrombocytopenia in advanced HIV disease. Megakaryocytes from HIV-1infected CD34+ progenitor cells are defective in their ability to produce platelets, as evidenced by hypercellular bone marrows with normal or increased numbers of megakaryocytes. These findings suggest that increased viral replication in the bone marrow microenvironment may cause this impairment and contribute to HIV-induced thrombocytopenia. . $^{8-10}$

\section{Management of thrombocytopenia in HIV}

Thrombocytopenia in HIV is rarely associated with bleeding and, for patients with platelet counts greater than $50 \mathrm{x}$ $10^{9} / \mathrm{L}$, no treatment is required. For some patients with clinically significant thrombocytopenia, short-term steroids produce a rapid response, but this has to be weighed against long-term infectious complications, as the steroids are immunosuppressive. Both antiretroviral agents and steroids improve thrombocytopenia by increasing platelet production without significantly affecting platelet survival., ${ }^{8,11,12}$

\section{Evans syndrome}

Patients can sometimes present with a combination of ITP and autoimmune haemolytic anaemia (AIHA). This http://dx.doi.org/10.4314/mmj.v27i3.8 
condition is known as Evans syndrome. Evans syndrome is defined by the combination of (either simultaneously or sequentially) autoimmune haemolytic anaemia and immune thrombocytopenia, sometimes together with immune neutropenia, in the absence of a known underlying cause. True Evans syndrome is a diagnosis of exclusion and other confounding disorders should not be present. ${ }^{13}$

A patient with Evans syndrome may present with the usual features of thrombocytopenia, such as petechiae, bruising, nose bleeding, gum bleeding, or menorrhagia; with or without features of haemolytic anaemia, such as jaundice, pallor and, in severe cases, heart failure. ${ }^{13}$

A full blood count of a patient with Evans syndrome will reveal the presence of cytopenias and a blood film examination will reveal features of haemolysis, such as red cell spherocytosis and polychromasia.

\section{Management of ITP and Evans syndrome}

The first-line therapy for ITP and Evans syndrome is corticosteroids. Prednisolone at a daily dose of 1 to $2 \mathrm{mg} / \mathrm{kg}$ is generally used. Patients generally respond when treated with prednisolone, however the cytopenias may recur on tapering or cessation of the steroids. ${ }^{13-16}$

For the patients in whom the conventional treatment with standard-dose corticosteroids is inappropriate, there are a wide variety of medical therapies. These include: (i) ciclosporin at a dose of $5 \mathrm{mg} / \mathrm{kg}$; (ii) cyclophosphamide at a dose of 1 to $2 \mathrm{mg} / \mathrm{kg}$; (iii) azathioprine at a dose of 1 to $2.5 \mathrm{mg} / \mathrm{kg} /$ day; (iv) oral high-dose dexamethasone $40 \mathrm{mg}$ daily for four days, repeated every 28 days for six cycles; (vi) parenteral methyl prednisolone, $30 \mathrm{mg} / \mathrm{kg} /$ day for three days, then $20 \mathrm{mg} / \mathrm{kg} /$ day for four days, then tapering to 5,2 , and $1 \mathrm{mg} / \mathrm{kg} /$ day each for one week; (vii) danazole at a dose of $200 \mathrm{mg}$ two to four times; (viii) high-dose intravenous immunoglobulin, IVIG, at a dose of $1 \mathrm{~g} / \mathrm{kg} / \mathrm{day}$; (ix) anti-CD20 monoclonal antibody, rituximab, at a dose of $375 \mathrm{mg} / \mathrm{m}^{2}$ and (x) intravenous anti-D, for RhD-positive patients who have not had splenectomy, at a dose of 50 to $75 \mu \mathrm{g} / \mathrm{kg}$. In ITP, anti-D is believed to work by preferentially destroying $\mathrm{RhD}$-positive red cells through the spleen, thus sparing autoantibody-coated platelets. ${ }^{15,17,18}$

TPO receptor agonists, such as romiplostim and eltrombopag (both are licenced for treatment of ITP) cause an increase in platelet count in ITP. One would expect that newly produced platelets, stimulated by TPO receptor agonists, would be rapidly destroyed by autoantibodies. It is believed that increased thrombopoiesis overcomes the autoantibodies; similarly, high doses of platelet transfusion can temporarily increase platelet count in ITP.

Splenectomy is traditionally used as a second-line therapy in patients who have failed to respond or relapsed following medical therapy with steroids. ${ }^{13-15}$

In Helicobacter pylori-associated ITP, eradication of $H$. pylori leads to a significant increase in platelet count. The association between $H$. pylori and ITP is likely related to molecular mimicry, whereby the antibodies produced against the pathogens react with antigens on the surface of platelets. ${ }^{4}$ In the acute setting, platelet and red cell transfusions may be required to alleviate symptoms, but their use should be minimized. ${ }^{13}$ The decision to transfuse platelets should be based on clinical and laboratory findings. Platelets are transfused to prevent or treat bleeding caused by platelet dysfunction. The total dose for platelets is 1 unit per $10 \mathrm{~kg}$, and this total dose should be given rapidly over 15 to 30 minutes. $^{19}$

\section{Childhood ITP and its management}

The diagnosis of childhood ITP is by exclusion. It can occur at anytime of childhood, but in the neonatal period, it must be distinguished from maternal ITP or alloimmune thrombocytopenia. ${ }^{18}$

In acute symptomatic ITP, the history is short with appearance of bruising and purpura within 24 to 48 hours, and the platelet count is usually less than $20 \times 10^{\circ} / \mathrm{L}$. This acute symptomatic ITP can be provoked by an acute viral infection or by immunization by the measles, mumps, and rubella (MMR) vaccine. ${ }^{18}$

ITP in children is uncommon and is usually transient. Childhood ITP is usually a benign disorder that requires no active management and serious bleeding is rare. About $80 \%$ of the children will recover spontaneously within six to eight weeks. ${ }^{18,20}$

For children who require treatment because of severe bleeding or to reduce the risk of severe bleeding, the standard firstline therapy in all the guidelines and consensus documents remains steroids. There is no consensus on dosing schedule, with some groups recommending a short course of highdose prednisolone, 3 to $4 \mathrm{mg} / \mathrm{kg} /$ day for a maximum of four days and others recommending conventional doses of 1 to $2 \mathrm{mg} / \mathrm{kg} /$ day for a maximum of 14 days. ${ }^{18,20}$

\section{Thrombocytopenia in pregnancy}

Thrombocytopenia occurs in $7 \%$ to $10 \%$ of pregnancies. Diagnostic and management difficulties often arise in this context. Aetiologies include gestational thrombocytopenia $(75 \%)$, thrombocytopenia secondary to hypertensive disorders $(20 \%)$, thrombocytopenia as an immune process (4\%), and others (1\%). ${ }^{15,21}$ The diagnosis of ITP involves exclusion of other causes of thrombocytopenia during pregnancy and normally does not involve bone marrow examination.

\section{Management of ITP in pregnancy}

Antenatal management of ITP depends on the extent of thrombocytopenia. Bleeding episodes caused by thrombocytopenia are less common in pregnant women than in non-pregnant women, as pregnancy is a hypercoagulable state, associated with increased levels of fibrinogen, factor VIII, and von Willebrand factor (vWF). There is also suppressed fibrinolysis and reduction in the activity of protein S, which is a natural anticoagulant. ${ }^{15,21}$

The aim of ITP management in pregnancy is to achieve and maintain "safer" rather than normal platelet counts. In the first two trimesters, asymptomatic patients with platelet counts greater than 20 to $30 \times 10^{\circ} / \mathrm{L}$ do not need any treatment until the third trimester. Treatment is required if platelet counts fall below 20 to $30 \times 10^{9} / \mathrm{L}$, when the patient is symptomatic, or when there is a need to increase platelet count to a level considered safe for a procedure. A platelet count of $50 \times 10^{9} / \mathrm{L}$ is usually safe for a procedure.

Antenatal full blood counts can be checked monthly until the third trimester and then two-weekly, then weekly as term approaches. $^{21}$

For those who require treatment, the usual first-line treatment is prednisolone, but at much lower doses: 20 to $30 \mathrm{mg}$ per day for a week. Although prednisolone is metabolized in the placenta by 11-beta-hydroxylase, high doses may have 
adverse effects on the pregnancy or foetus, including premature rupture of membranes, adrenal suppression, and a slightly increased risk of cleft lip after use in the first trimester. Platelet counts may fall more rapidly near term, so defer tapering of steroids until after delivery. ${ }^{15,21}$

The mode of delivery should be based on obstetric considerations. From 35 to 36 gestational weeks, or prior to any intervention, give treatment to raise platelet counts to at least $50 \times 10^{9} / \mathrm{L}$ and aim to attain a platelet count of more than $80 \times 10^{9} / \mathrm{L}$ to allow for regional anaesthesia, as many anaesthetists would not consider an epidural below this level. ${ }^{15}$

Where the maternal platelet count remains below $50 \times 10^{9} / \mathrm{L}$ around the time of delivery, platelets for transfusion should be available on standby. Platelets should be transfused during well-established labour and if there are increased bleeding complications. $^{15}$

\section{Differential diagnoses of thrombocytopenia}

As stated earlier, ITP is just one of the causes of thrombocytopenia. When faced with a patient with thrombocytopenia, it is important to know that there are other possible causes. The other causes of thrombocytopenia include: (i) infections such as malaria and sepsis; (ii) disseminated intravascular coagulation (DIC); (iii) thrombotic thrombocytopenic purpura/haemolytic uremic syndrome (TTP/HUS); and (iii) heparin-induced thrombocytopenia (HIT).

ITP can sometimes be diagnosed incidentally if the patient does not have mucocutaneous bleeding, while TTP/HUS is a haematological emergency, with patients presenting critically ill.

\section{Thrombotic thrombocytopenic purpura/haemolytic uraemic syndrome (TTP/HUS)}

Thrombotic thrombocytopenic purpura and haemolytic uraemic syndrome are two different disorders with disparate pathogenetic mechanisms; however, they have very similar presentations and thus are grouped into the single designation TTP/HUS. HUS is caused by Shiga toxin-producing strains of Escherichia coli and Shigella dysenteriae. TTP has a predilection for young females in their thirties with advanced $\mathrm{HIV}$ and is less frequent in those on antiretroviral therapy. Novitzky et al. reported that patients with HIV-associated TTP were exclusively of the African Black race and that they were mainly women. ${ }^{22}$ It is caused by $\mathrm{IgG}$ autoantibodies to a vWF-cleaving enzyme called ADAMTS13 (a disintegrin-like metalloprotease with thrombospondin type 1 motif 13$){ }^{22-25}$ T'TP and HUS are characterised by the formation of thrombi in the arterioles and capillaries and a pentad of symptoms and signs, which are thrombocytopenia, microangiopathic haemolytic anaemia, neurologic abnormalities, renal failure, and fever. Coombs test is negative. In TTP, thrombocytopenia and haemolysis are caused by clumping of platelets and shearing of red cells by mechanical damage, while in HUS they are caused by the direct cytotoxic effects of Shiga toxin and complement activation.

\section{Management of TTP/HUS}

Plasmapheresis (plasma exchange) is the standard treatment of T'TP, as it removes the autoantibodies against the vWFcleaving enzyme and replaces the fresh enzyme. This treatment requires expensive equipment and skilled operators, both of which are not available in most developing countries, including Malawi. Fresh frozen plasma (FFP) infusions at a dose of $30 \mathrm{~mL} / \mathrm{kg} /$ day ( \pm diuresis with frusemide) in three divided doses (provided the patient can tolerate the volume load) can be effective. ${ }^{22}$

\section{Heparin-induced thrombocytopenia (HIT)}

Heparin-induced thrombocytopenia is a potentially serious drug adverse effect more frequently associated with unfractionated heparin (UFH) than low molecular weight heparin (LMWH). Heparin induces platelet activation and aggregation and activates the coagulation system. HIT does not usually cause bleeding, but instead causes thrombosis. ${ }^{26}$ Management of HIT includes withdrawing the heparin and the use of an alternative anticoagulant, such as streptokinase, when acute venous or arterial thrombosis is present. ${ }^{26}$ In the presence of severe or extending thrombosis, commencement of warfarin should be delayed for a few days, as protein $\mathrm{C}$ and protein $\mathrm{S}$ reduction induced by warfarin can lead to thrombus progression. ${ }^{26}$

\section{Disseminated intravascular coagulation (DIC)}

Disseminated intravascular coagulation (DIC) never occurs in isolation, and it is associated with a range of illnesses and conditions. These include sepsis, trauma, malignancy, ABO transfusion incompatibility, and obstetric and gynaecological conditions, such as amniotic fluid embolism, placental abruption, and pre-eclampsia. ${ }^{27}$ DIC is characterised by generalised intravascular activation of haemostatic mechanisms, leading to widespread fibrin clot formation, consumption of platelets and coagulation factors, and activation of fibrinolysis. ${ }^{27}$

\section{Diagnosis of disseminated intravascular coagulation}

Diagnosis of DIC requires clinical and laboratory information. The patient should have an underlying condition known to be associated with a DIC. Laboratory tests should include full blood count (to note the trend of platelet reduction: a continuous drop in platelet count, even if the count is within the normal range of 150 to $400 \times 10^{9} / \mathrm{L}$, may indicate DIC); prothrombin time (PT), and activated partial thromboplastin time (aPT'), which are prolonged in the context of increased consumption of coagulation factors. ${ }^{27,28}$

The International Society on Thrombosis and Haemostasis (ISTH) recommends the use of scoring systems for overt DIC and non-overt DIC. ${ }^{29}$

Scoring system for overt DIC:

1.Risk assessment: Does the patient have an underlying disorder known to be associated with overt DIC?
If yes: proceed
If not: do not use this algorithm

2. Order global coagulation tests (platelet count, PT, fibrinogen, fibrin-related marker)

3.Score the global coagulation test results

- Platelet count $(>100=0 ;<100=1 ;<50=2)$

- Prolonged PT $(<3 \mathrm{~s}=0 ;>3$ but $<6 \mathrm{~s}=1 ;>6 \mathrm{~s}=2)$

- Elevated fibrin marker (e.g., D-dimer, fibrin degradation products) (no increase $=0$; moderate increase $=1$; strong increase $=2$ )

- Fibrinogen level $(>1=0 ;<1=1)$

\section{Calculate score:}

- Scores $\geq 5$ are compatible with overt DIC: repeat score daily

- Scores $<5$ are suggestive of non-overt DIC: repeat next 1 to 2 days

http://dx.doi.org/10.4314/mmj.v27i3.8 


\section{References}

1. Hoffbrand AV, Pettit JE, Moss PAH. Essential haematology. 4th ed. Williston, Vermont, USA: Wiley-Blackwell; 2002.

2. Lichtman MA, Beutler E, Kaushansky K, Kipps TJ, Seligsohn U, Prchal J. Williams hematology. 7th ed. New York: McGraw-Hill; 2005.

3. Cines DB, McMillan R. Pathogenesis of chronic immune thrombocytopenia. Curr Opin Hematol. 2007;14(5):511-4.

4. Cooper N, Bussel J. The Pathogenesis of immune thrombocytopenia. Br J Haematol. 2006;133(4):364-74.

5. Cines DB, Bussel J, Liebman HA, Luning Prak ET. The ITP syndrome: pathogenesis and clinical diversity. Blood. 2009 Jun 25;113(26):6511-21. doi: 10.1182/blood-2009-01-129155.

6. Nugent D, McMillan R, Nichol JL, Slichter SJ. Pathogenesis of chronic immune thrombocytopenia: increased platelet destruction and or decreased platelet production. Br J Haematol. 2009 Sep;146(6):58596. doi: 10.1111/j.1365-2141.2009.07717.x.

7. Toltl LJ, Arnold DM. Pathophysiology and management of chronic immune thrombocytopenia: focusing on what matters. Br J Haematol. 2011 Jan;152(1):52-60. doi: 10.1111/j.1365-2141.2010.08412.x.

8. Sloand E. Haematologic complications of HIV infection. AIDS Reviews. 2005;7:187-96.

9. Sloand EM, Klein HG, Banks SM, Vareldzis B, Merrit S, Pierce P. Epidemiology of thrombocytopenia in HIV infection. Eur J Haematol. 1992 Mar;48(3):168-72.

10. Zauli G, Re M, Davis B, Sen L, Visani G, Gugliotta L, et al. Impaired in vitro growth of purified $(\mathrm{CD} 34+)$ haematopoietic progenitors in HIV-1 seropositive thrombocytopenic individuals. Blood. 1992 May 15;79(10):2680-7.

11. Gernsheimer T, Stratton J, Ballem PJ, Slichter SJ. Mechanisms of Response to Treatment in Autoimmune Thrombocytopenic Purpura. N Engl J Med. 1989 Apr 13;320(15):974-80.

12. Ballem PJ, Belzberg A, Devine DV, Lyster D, Spruston B, Chambers $\mathrm{H}$, et al. Kinetic studies of the mechanism of thrombocytopenia in patients with Human Immunodeficiency Virus infection. N Engl J Med. 1992 Dec 17;327(25):1779-84.

13. Norton A, Roberts I. Management of Evans syndrome. Br J Haematol. 2006 Jan;132(2):125-37.

14. McVerry BA. Management of idiopathic thrombocytopenic purpura. Br J Haematol. 1985 Feb;59(2):203-8.

15. Myers B. Diagnosis and management of maternal thrombocytopenia in pregnancy. Br J Haematol. 2012 Jul;158(1):3-15. doi: 10.1111/j.13652141.2012.09135.x.

16. Ghanima W, Godeau B, Cines DB and Bussel BJ. How I treat immune thrombocytopenia: the choice between splenectomy or a medical therapy as a second-line treatment. Blood. 2012 Aug 2;120(5):960-9. doi: 10.1182/blood-2011-12-309153.
17. Gibbon, CJ, editor. South African medicines formulary. 6th ed. Pinelands, South Africa: The South African Medical Association Health and Medical Publishing Group. 2003.

18. British Committee for Standards in Haematology General Haematology Task Force. Guidelines for the investigation and management of idiopathic thrombocytopenic purpura in adults, children and in pregnancy. Br J Haematol. 2003 Feb;120(4):574-96.

19. Chosamata BI, M'baya B, Njolomole S. Guidelines for the clinical use of blood and blood products in Malawi. 1st ed. Lilongwe: Ministry of Health, Malawi; 2012.

20. Cooper N. A review of the management of childhood immune thrombocytopenia: how can we provide an evidence-based approach? Br J Haematol. 2014 Jun;165(6):756-67. doi: 10.1111/bjh.12889.

21. Provan D, Stasi R, Newland AC, Blanchette VS, Bolton-Maggs P, Busse JB, et al. International consensus report on the investigation and management of primary immune thrombocytopenia. Blood. 2010 Jan 14;115(2):168-86. doi: 10.1182/blood-2009-06-225565.

22. Novitzky N, Thomson J, Abrahams L, du Toit C, McDonald A. Thrombotic thrombocytopenic purpura in patients with retroviral infection is highly responsive to plasma infusion therapy. Br J Haematol. $2005 \mathrm{Feb}$;128(3):373-9.

23. Levy GG, Motto DG, Ginsburg D. ADAMTS13 turns 3. Blood. 2005 Jul 1;106(1):11-7.

24. Derad I, Obermann B, Katalinic A, Eisemann N, Knobloch JK, Sayk F, et al. Hypertension and mild chronic kidney disease persist following severe haemolytic uraemic syndrome caused by Shiga toxin-producing Escherichia coli O104:H4 in adults. Nephrol Dial Transplant. 2015 Jul 14;0:1-9. doi: 10.1093/ndt/gfv255

25. Hwang WY, Chai LY, Ng HJ, Goh YT, Tan PH. Therapeutic plasmapheresis for the treatment of the thrombotic thrombocytopenic purpura-haemolytic uraemic syndromes. Singapore Med J. 2004 May;45(5):219-23.

26. Chong BH. Heparin-induced thrombocytopenia. J Thromb Haemost. $2003 \mathrm{Jul} ; 1(7): 1471-8$

27. Levi M, Toh $\mathrm{CH}$, Thachil J, Watson HG; British Committee for Standards in Haematology. Guidelines for the diagnosis and management of disseminated intravascular coagulation. Br J Haematol. 2009 Apr;145(1):24-33. doi: 10.1111/j.1365-2141.2009.07600.x.

28. Provan D, Singer CRJ, Baglin T and Lilleyman J, eds. Oxford handbook of clinical hematology. 2004, Oxford University Press.

29. Toh $\mathrm{CH}$, Hoots WK; SSC on Disseminated Intravascular Coagulation of the ISTH.. The scoring system of the scientific and standardisation committee on disseminated intravascular coagulation of the International Society on Thrombosis and Haemostasis: a 5-year overview. J Thromb Haemost. 2007 Mar;5(3):604-6. 